UNIVERSIDADE DE BRASÍLIA

FACE / DEPARTAMENTO DE ECONOMIA

PROGRAMA DE PÓS-GRADUAÇÃO EM ECONOMIA

MARCELO CRUZ DE SOUZA

ESCOLHAS ALEATÓRIAS COM CATEGORIAS

Dissertação apresentada como requisito parcial para obtenção do título de Mestre em Economia pelo Programa de Pós-Graduação em Economia da Universidade de Brasília

Orientador: Prof. Gil Riella

Brasília 
MARCELO CRUZ DE SOUZA

\title{
ESCOLHAS ALEATÓRIAS COM CATEGORIAS
}

\begin{abstract}
Dissertação apresentada como requisito parcial para obtenção do título de Mestre em Economia pelo Programa de Pós-Graduação em Economia da Universidade de Brasília
\end{abstract}

Aprovado em 7 de dezembro de 2015

BANCA EXAMINADORA

Gil Riella (presidente)

Universidade de Brasília

Leandro Gonçalves do Nascimento

Universidade de Brasília

José Guilherme de Lara Resende (suplente)

Universidade de Brasília 


\section{Resumo}

Caracterizamos um modelo de escolhas aleatórias em que o tomador de decisão classifica seu universo de opções em categorias e tem preferências completas e transitivas dentro de cada categoria. Em face de um problema de escolha, uma categoria é sorteada segundo uma função de probabildade, e ele maximiza sua preferência dentro da categoria considerada. $\mathrm{O}$ comportamento aleatório resulta do sorteio da cateogira a ser considerada. Este modelo estende a ideia central de Furtado et al (2015), focado em escolhas determinísticas, ao paradigma das escolhas aleatórias desenvolvido de forma pioneira por Luce (1959). É um caso particular do modelo geral de Maximização Randômica de Utilidade criado por Block e Marschak (1959) e Marshak (1960) e caracterizado por Falmagne (1978) e Barberá e Pattanaik (1986), e mais geral que o modelo de Manzini e Mariotti (2014). 


\begin{abstract}
We characterize a model of random choices in which the decision maker classifies her universe in categories and has complete and transitive preferences in each category. When faced with a decision problem, she sorts one category according to a probability function, and maximizes her preference in the sorted category. The random behavior results from the sorting of the category to be considered. This model extends the central idea of Furtado et al (2015), which is focused in deterministic, to the paradigm of the random choices pioneered by Luce (1959). It is a special case of the general model of Random Utility Maximization introduced into Economics by Block and Marschak (1959) and Marshack (1960) and characterized by Falmagne (1978) and Barberá and Pattanaik (1986), and is more general than Manzini and Mariotti (2014).
\end{abstract}


À minha esposa Talita Aos meus filhos Davi, Marcela, Isabela e Giovana 


\section{AGRADECIMENTOS}

A Deus pelos dons que me concedeu, pela minha família, meus amigos, meu trabalho e pelas oportunidades de aprendizado com que me agraciou.

À minha família, pela paciência e pelo apoio durante esse projeto.

Ao Gil, pelas aulas, pelos insights, pelos desafios e pela orientação acadêmica ao longo desses dois anos.

Aos meus professores, dos quais, além do Gil, gostaria de mencionar, sem excluir os demais, o Leandro e o José Guilherme, por tudo que me ensinaram.

À minha turma, especialmente Maurício, Felipe e Rafael, verdadeiros amigos e também pacientes professores.

Ao Banco Central do Brasil pelo suporte através do Programa de Pós-Graduação, e à Universidade de Brasília, pelo curso ofertado. 


\section{Sumário}

1 Introdução $\quad 8$

2 Formalização do modelo $\quad 9$

3 Resultados $\quad \mathbf{1 1}$

3.1 Modelo geral . . . . . . . . . . . . . . . . . . . 12

3.2 MM - caso particular de Categorias . . . . . . . . . . . . . 13

3.3 Categorias disjuntas . . . . . . . . . . . . . . . . 14

4 Conclusão 15

A Complemento das demonstrações $\mathbf{1 5}$

A.1 Teorema 1...................... 15

A.2 MM - caso especial . . . . . . . . . . . . . . . . 22

A.3 Teorema $2 \ldots \ldots \ldots \ldots$ 


\section{Introdução}

Furtado et al. [2015] (FNR) propõem um modelo de escolha em que o mecanismo cognitivo da categorização, isto é, a divisão do universo de opções em categorias, com preferências completas e transitivas em cada categoria, cumpre papel central no processo de decisão $\mathrm{O}$ modelo oferece uma convincente explicação para padrões de decisão que desviam do Axioma Fraco da Preferência Revelada de Samuelson [1938], em consequência de limitações comportamentais, cognitivas e de atenção. Seguindo a teoria da escolha clássica, pressupõe-se o comportamento determinístico: dado um problema de escolha, a resposta do tomador de decisão é um subconjunto dos itens do problema de escolha, sem indicação de frequência ou ordem entre os itens escolhidos em um mesmo problema de escolha.

Diferentemente do modelo clássico de escolha determinística, no presente trabalho a decisão do agente é revelada por uma probabilidade: nossa premissa é uma regra que associa a cada opção de um problema de escolha a probabilidade de que esta seja escolhida, conforme introduzido por Luce [1959], Block and Marshak [1960], Marshak [1960] e desenvolvido recentemente por Gul et al. [2014], Manzini and Mariotti [2014]. A definição de racionalidade proposta por Falmagne [1978], Barberá and Pattanaik [1986] caracteriza o modelo mais geral de escolhas aleatórias, o Random Utility Maximization (RUM), inicialmente proposto por Block and Marshak [1960], Marshak [1960]. No modelo de escolhas aleatórias desenvolvido em Manzini and Mariotti [2014] (MM), o indivíduo revela uma preferência sobre o universo de opções - uma opção é revelada (fracamente) preferível a outra se a presença da primeira em algum problema de escolha reduz a probabilidade de que a segunda seja escolhida - e a aleatoriedade na escolha resulta de um componente de atenção. Dado um problema de escolha, um subconjunto do universo é sorteado para ser considerado, conforme a probabildade de cada opção, e o indivíduo maximiza sua preferência subjacente, revelada pelo padrão de escolha. No modelo desenvolvido aqui, a preferência é revelada como em MM, e a cada categoria está associada uma probabilidade de que esta seja sorteada quando da decisão referente a um problema de escolha.

A categorização pode ser interpretada como uma limitação de atenção por incapacidade de considerar todo o universo de opções. Pode-se também pensar nas categorias como a representação de atributos: cada categoria é associada a um atributo e engloba as opções que o têm, e, dado um problema de escolha, o universo de opções é limitado às opções que têm o atributo em foco. O seguinte 
exemplo ilustra a flexibilidade e o poder descritivo do modelo de escolha aleatória por categorias: Suponha que Ana escolhe diariamente um meio de transporte público para ir ao trabalho. Na cidade há taxis e ônibus, cada tipo podendo ser climatizado ou não. Em $10 \%$ dos dias, Ana precisa usar taxi, e em $20 \%$ dos dias, ela precisa usar transporte climatizado, não havendo correlação entre essas necessidades. A oferta de transporte varia a cada dia por razões diversas. Desde que sua necessidade seja atendida, Ana escolhe o transporte mais barato - na ordem, ônibus comum, ônibus climatizado, taxi comum, taxi climatizado; e se sua necessidade não for atendida, Ana desiste de usar transporte público. Este é um exemplo bastante razoável de padrão de decisão que, como veremos adiante, pode ser representado de forma simples e intuitiva em um modelo de categorização. Apesar da flexibilidade e do poder descritivo da categorização, não se encontra na literatura, que seja do nosso conhecimento, um modelo que represente esse padrão de decisão da forma aqui proposta.

A seção 2 a seguir apresenta os conceitos fundamentais da teoria da escoha aleatória e formaliza matematicamente o modelo de escolha por categorias, a seção 3 mostra os resultados e a seção 4 faz a conclusão.

\section{Formalização do modelo}

O universo de opções disponíveis ao agente decisor é representado pelo conjunto $X$. Um campo de escolha sobre $X$, simbolizado por $\Omega$, é uma família de subconjuntos de $X$ fechada para uniões finitas. Este campo de escolha é interpretado como o conjunto dos "menus", ou problemas de escolha. Quando $|X| \geqslant 3$, referimo-nos ao par $(X, \Omega)$ como um espaço de escolha. O espaço de escolha é dito finito quando $X$ é finito. (Por simplicidade, no presente trabalho consideramos que $X$ é finito, $|X| \geqslant 3$ e $\Omega=2^{X} \backslash \emptyset$.)

Dados um problema de escolha e uma opção, observa-se a frequência com que o agente escolhe aquela opção. Como em MM, permite-se que o agente não escolha nada, mas aqui se faz exceção ao menu completo, $X$, no qual o agente necessariamente escolhe alguma opção. Isso se reflete na possibilidade de que a soma das probabilidades das opções em um mesmo menu seja inferior a 1, exceto no menu $X$. Formalmente:

Definição 1. Uma regra de escolha aleatória é uma função $\sigma: \Omega \times X \rightarrow[0,1]$ satisfazendo $\sum_{x \in X} \sigma(X, x)=1, \sum_{x \in A} \sigma(A, x) \leq 1$ e $\sigma(A, x)=0 \forall x \notin A$. Para dados $A \in \Omega$ e $x \in A, \sigma(A, x)$ é interpretado como a probabilidade ou a 
frequência com que o agente escolhe a opção $x$ quando deparado com o problema de escolha $A$.

A probabilidade de não escolher nada é representada pelo parâmetro null: denotamos $\sigma(A$, null $)=1-\sum_{x \in A} \sigma(A, x)$. Denotamos ainda $p_{x}=\sigma(\{x\}, x)$.

Estabelecemos o conceito de categorização conforme FNR.

Definição 2. Dada uma ordem parcial estrita (assimétrica e transitiva) $\succ$ sobre $X$, uma categorização de $X$ é uma cobertura $\mathcal{S}$ de $X$ tal que $\succ$ é completa em cada $S \in \mathcal{S}$. A categorização é dita disjunta se $\mathcal{S}$ é uma partição de $X$. Qualquer $S \in \mathcal{S}$ é chamado uma categoria de $\mathcal{S}$, e é não vazio.

O conceito de escolhas por categorias, central para o presente trabalho, é definido a seguir.

Definição 3. Uma regra de escolha aleatória $\sigma$ é uma regra de escolha por categorias se existem uma ordem parcial estrita $\succ$ sobre $X$, uma categorização $\mathcal{S}$ de $X$ associada a $\succ$ e uma função de probabilidade $\pi: \mathcal{S} \rightarrow[0,1]$ com $\sum_{S \in \mathcal{S}} \pi(S)=1$ tais que, para todo menu $A \in \Omega$,

$\sigma(A, x)=\left\{\begin{array}{l}0, \text { se } x \notin A \\ \sum\{\pi(S): S \in \mathcal{S}, x \in S \text { e } x \succ y \text { para todo } y \in[A \cap S] \backslash\{x\}\}, \text { se } x \in A\end{array}\right.$

Dizemos que $\sigma$ é uma regra de escolha por categorias representada por $(\succ$ $, \mathcal{S}, \pi)$

Consideraremos ainda o caso especial em que a escolha é explicada por uma categorização disjunta:

Definição 4. Uma regra de escolha aleatória $\sigma$ é uma regra de escolha por categorias disjuntas se $\mathcal{S}$ é uma categorização disjunta. Nesse caso, $\sigma$ pode ser descrita por

$$
\sigma(A, x)=\left\{\begin{array}{l}
0, \text { se } x \notin A \text { ou } \exists y \in S_{x} \cap A \text { tal que } y \succ x \\
\pi\left(S_{x}\right), \text { caso contrário }
\end{array}\right.
$$

em que $S_{x} \in \mathcal{S}$ é a categoria a que $x$ pertence.

Podemos agora formalizar o exemplo apresentado na introdução.

Exemplo 1. O universo de opções é dado por $X=\{o, O, t, T\}$, onde $o, O$, $t$ e $T$ representam, respectivamente, ônibus comum, ônibus climatizado, taxi 
comum e taxi climatizado. Os menus são todos os subconjuntos não vazios de $X$, isto é, $\Omega=2^{X} \backslash \emptyset$. O agente divide seu universo nas categorias $\{T\}$, $\{t, T\},\{T, O\}$ e $\{t, T, o, O\}$, com respectivas probabilidades $2 \%$ (necessidade de usar taxi climatizado), $18 \%$ (necessidade de usar taxi), $8 \%$ (necessidade de usar transporte climatizado) e os restantes $72 \%$. Em uma mesma categoria, a preferência é dada por $o \succ O \succ t \succ T$. Em face de um problema de escolha (um elemento de $\Omega$ ), o agente sorteia uma categoria e escolhe seu $\succ-$ máximo. A regra de escolha aleatória resultante é mostrada na tabela abaixo.

\begin{tabular}{|c|c|c|c|c|}
\hline \multirow[b]{2}{*}{ Menu } & \multicolumn{4}{|c|}{ Opçāo / probabilidade de escolha } \\
\hline & 0 & 0 & $t$ & $T$ \\
\hline$o$ & $72 \%$ & - & 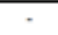 & 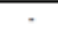 \\
\hline$O$ & . & $80 \%$ & - & - \\
\hline$t$ & - & $\cdot$ & $90 \%$ & - \\
\hline$T$ & . & - & - & $100 \%$ \\
\hline 00 & $32 \%$ & $8 \%$ & . & . \\
\hline ot & $72 \%$ & $\cdot$ & $18 \%$ & - \\
\hline oT & $72 \%$ & - & - & $28 \%$ \\
\hline$O t$ & - & $80 \%$ & $20 \%$ & - \\
\hline OT & - & $80 \%$ & - & $20 \%$ \\
\hline$t T$ & - & - & $92 \%$ & $8 \%$ \\
\hline$O O t$ & $72 \%$ & $10 \%$ & $18 \%$ & - \\
\hline OOT & $72 \%$ & $8 \%$ & - & $20 \%$ \\
\hline ot $T$ & $72 \%$ & - & $18 \%$ & $10 \%$ \\
\hline OtT & - & $80 \%$ & $18 \%$ & $2 \%$ \\
\hline OOTT & $72 \%$ & $8 \%$ & $18 \%$ & $2 \%$ \\
\hline
\end{tabular}

O objetivo central deste trabalho é a caracterização axiomática das regras de escolha aleatória que resultam de um processo de escolha por categorização. Em outras palavras, respondemos à questão: de posse de informações sobre um padrão de decisão revelado por frequências de escolha de opções em menus, que propriedades necessárias e suficientes devem ser testadas a fim de avaliar se esse padrão de decisão pode ser descrito pelo modelo de escolha por categorias aqui exposto. Também são analisadas as condições de unicidade e a relação com outros modelos da literatura.

\section{Resultados}

Definimos a relação $\triangleright \subseteq X \times X$ por $x \triangleright y$ se para algum $A \in \Omega \sigma(A \cup\{x\}, y)<$ $\sigma(A, y)$.

Dada uma ordem parcial estrita $\succ$, denotamos $L_{\succ}(x)=\left\{x^{\prime} \in X: x \succ x^{\prime}\right\}$ e $U_{\succ}(x)=\left\{x^{\prime} \in X: x^{\prime} \succ x\right\}$. Esta seção traz apenas a parte central das demonstrações, cujos detalhes são dados no apêndice. 


\subsection{Modelo geral}

Usamos uma função auxiliar semelhante àquela definida em Falmagne [1978], Barberá and Pattanaik [1986]. Dada uma regra de escolha aleatória $\sigma$, define-se a função $q_{\sigma}: 2^{X} \rightarrow \mathbb{R}$ recursivamente da seguinte forma.

$$
\begin{aligned}
q_{\sigma}(\emptyset, x) & =\sigma(X, x) \\
\text { Para } S \neq \emptyset, q_{\sigma}(S, x) & =\left\{\begin{array}{l}
0, \text { se } x \in S \\
\sigma(X \backslash S, x)-\sum_{S^{\prime} \subset S} q_{\sigma}\left(S^{\prime}, x\right), \text { se } x \notin S
\end{array}\right. \\
q_{\sigma}(\emptyset, \text { null }) & =\sigma(X, \text { null })=0 \\
q_{\sigma}(S, \text { null }) & =\sigma(X \backslash S, \text { null })-\sum_{S^{\prime} \subset S} q_{\sigma}\left(S^{\prime}, \text { null }\right)
\end{aligned}
$$

Por consistência da fórmula com o domínio de $q_{\sigma}$, consideramos que $\sigma(\emptyset, x)=$ 0 e $\sigma(\emptyset, n u l l)=1$. O seguinte axioma impõe a mesma condição de Falmagne [1978], Barberá and Pattanaik [1986].

Axioma 1. (Racionalidade Estocástica) Para todos $S \in 2^{X}$ e $x \in X q_{\sigma}(S, x) \geq$ 0 e $q_{\sigma}(S$, null $) \geq 0$.

Acrescentamos a condição de aciclicidade.

Axioma 2. (Aciclicidade) A relação $\triangleright$ é acíclica.

E obtemos nosso principal resultado.

Teorema 1. Uma regra de escolha aleatória $\sigma$ é uma regra de escolha por categorias se e somente se $\sigma$ satisfaz aos axiomas de Racionalidade Estocástica e Aciclicidade. Além disso, a representação por categorias é única.

Demonstração. $[\Rightarrow]$ Seja $\sigma$ uma regra de escolha por categorias representada por $(\succ, \mathcal{S}, \pi)$. Pode-se verificar que para todos $S \in 2^{X}$ e $x \in X \backslash S$

$$
\begin{aligned}
q_{\sigma}(S, x) & =\left\{\begin{array}{l}
0 \text { se } S \cap L_{\succ}(x) \neq \emptyset \\
\sum_{R \subseteq L_{\succ}(x)} \pi(S \cup R \cup\{x\}) \geq 0 \text { caso contrário }
\end{array}\right. \\
q_{\sigma}(S, \text { null }) & =\pi(S) \geq 0
\end{aligned}
$$

Por outro lado, para a relação $\triangleright$ definida sobre $\sigma$, temos $\triangleright \subseteq \succ$. Como por hipótese $\succ$ é uma ordem parcial estrita, e portanto acíclica, $\triangleright$ também é acíclica. 
$[\Leftarrow]$ Considere agora uma regra de escolha aleatória $\sigma$ satisfazendo aos axiomas. Construímos uma representação de $\sigma$ na forma de uma regra de escolha por categorias.

Seja $n=|X|$. Nomeie os elementos de $X$ por $x_{1}, \ldots, x_{n}$ de forma que $i<j \Rightarrow$ $x_{i} \not x_{j}$. Para $k \in\{1, \ldots, n\}$, seja $\mathcal{S}^{(k)}=\left\{S \in 2^{X}: S \cap\left\{x_{k}, \ldots, x_{n}\right\}=\left\{x_{k}\right\}\right\}$. Obviamente, $\bigcup \mathcal{S}=2^{X} \backslash \emptyset$. Defina $\pi: 2^{X} \backslash \emptyset \rightarrow[0,1]$ da seguinte forma.

Para $k \in\{1, \ldots, n\}$ e $S \in \mathcal{S}^{(k)}$, faça $\pi(S)=q_{\sigma}\left(S \backslash\left\{x_{k}\right\}, x_{k}\right)-\sum_{i=k+1}^{n} q_{\sigma}\left(S, x_{i}\right)$. Teremos $\pi(S) \geq 0$.

Por fim, faça $\succ=\left\{\left(x_{j}, x_{i}\right): j>i\right\}$, e $\mathcal{S}=\left\{S \in 2^{X} \backslash \emptyset: \pi(S)>0\right\}$.

A construção acima garante que, para todo $A \in \Omega$ e todo $x \in A, \sigma(A, x)=$ $\sum_{S \subseteq[X \backslash A] \cup L_{\succ}(x)} \pi\left(S \cup\left\{x_{k}\right\}\right)$. Por fim, temos que $\sum_{S \in \mathcal{S}} \pi(S)=1$. Conclui-se que $\sigma$ é uma regra de escolha por categorias representada por $(\succ, \mathcal{S}, \pi)$.

[Unicidade] Dada uma representação de $\sigma(\succ, \mathcal{S}, \pi), \pi$ é determinada unicamente pela fórmula $\pi(S)=q_{\sigma}(S$, null $)$. E como $\sum_{S \in \mathcal{S}} \pi(S)=1$, concluimos que a representação é única.

\subsection{MM - caso particular de Categorias}

No modelo MM, uma regra de escolha aleatória $\sigma$ é uma regra aleatória por conjunto de consideração (random consideration set rule) se existem uma uma ordem total estrita $\succ$ sobre $X$ e uma função $\gamma: X \rightarrow(0,1)$ tais que para todos $A \in \Omega$ e $x \in A$

$$
\sigma(A, x)=\gamma(x) \prod_{y \in A: y \succ x}(1-\gamma(y))
$$

Se impusermos $\sum_{x \in X} \sigma(X, x)=1$ nesse modelo, fazendo $\mathcal{S}=2^{X} \backslash \emptyset$ e $\pi(S)=$ $\prod_{x \in S} \gamma(x) \prod_{y \notin S}(1-\gamma(y))$, teremos $\sigma(A, x)=\gamma(x) \prod_{y \in A \cap U_{\succ}(x)}(1-\gamma(y))$, donde se conclui que $\sigma$ é um caso particular de regra de escolha por categorias. Manzini and Mariotti [2014] caracterizam esse modelo por meio dos axiomas de i-Assimetria e i-Independência. Permanece como objeto de pesquisa o desenvolvimento do modelo MM a partir do de categorias, isto é, mantidos os axiomas de Racionalidade Estocástica e Aciclicidade, a análise das condições adicionais que o modelo MM impõe. 


\subsection{Categorias disjuntas}

Estabelecemos agora a condição necessária e suficiente para que uma regra de escolha aleatória possa ser caracterizada como uma regra de escolha por categorias disjuntas.

Axioma 3. (p-Independência) Se para algum $A \in \Omega_{X} \sigma(A, y) \neq \sigma(A \cup\{x\}, y)$ então para todo $B \in \Omega_{X} \sigma(B x, y)=0$ e $\sigma(B \cup\{x\}$, null $)=\sigma(B$, null $)$.

E chegamos ao nosso segundo resultado.

Teorema 2. Uma regra de escolha aleatória $\sigma$ é uma regra de escolha por categorias disjuntas se e somente se $\sigma$ satisfaz p-Independência.

Demonstração. $[\Rightarrow]$ Seja $\sigma$ uma regra de escolha por categorias disjuntas representada por $(\succ, \mathcal{S}, \pi)$. Sejam $x, y \in X$ tais que para algum $A \in \Omega_{X} \sigma(A, y) \neq$ $\sigma(A \cup\{x\}, y)$. Claramente, $x$ e $y$ são da mesma categoria e $x \succ y$, donde $\sigma(A \cup\{x\}, y)=0$ para todo $A \in \Omega$. Quanto à segunda implicação, seja $w=\max _{\succ}\left(S_{x}\right) \cap[A \cup\{y\}]$. Claramente, $\sigma(A \cup\{y\}, w)=\pi\left(S_{x}\right)$. Se $x \triangleright w$, tere$\operatorname{mos} \sigma(A \cup\{x, y\}, x)=\sigma(A \cup\{y\}, w), \sigma(A \cup\{x, y\}, w)=0$ e $\sigma(A \cup\{x, y\}, z)=$ $\sigma(A \cup\{y\}, z) \forall z \in[A \cup\{y\}] \backslash\{w\}$, donde segue que $\sigma(A \cup\{x, y\}$, null $)=\sigma(A \cup$ $\{y\}, n u l l)$. Por outro lado, se $x \not w$, como $x$ e $w$ são da mesma categoria, $w \succ x$ e portanto $\sigma(A \cup\{x, y\}, x)=0$ e $\sigma(A \cup\{x, y\}, z)=\sigma(A \cup\{y\}, z) \forall z \in[A \cup\{y\}]$, e novamente temos $\sigma(A \cup\{x, y\}$, null $)=\sigma(A \cup\{y\}$, null $)$.

$[\Leftarrow]$ Considere agora $\sigma$ satisfazendo p-Independência. Verificam-se os seguintes fatos.

1. Para todos $A \in \Omega$ e $x \in X \sigma(A, x)=\left\{\begin{array}{l}0, \text { se } x \notin A \text { ou } \exists y \in A: y \triangleright x \\ p_{x}, \text { caso contrário }\end{array}\right.$

2. (Monotonicidade) Para todo $A, B \in \Omega$ com $A \subseteq B, \forall x \in A \sigma(A, x) \geq$ $\sigma(B, x)$ e $\sigma(A$, null $) \geq \sigma(B$, null $)$.

3. (Transitividade) $x \triangleright y \triangleright z \Rightarrow x \triangleright z$.

4. $x \triangleright y \Rightarrow p_{x}=p_{y}$.

5. $x \triangleright y, z \Rightarrow y \triangleright z$ ou $z \triangleright y$.

6. $x, y \triangleright z \Rightarrow x \triangleright y$ ou $y \triangleright x$. 
Definimos a relação $\equiv \subseteq X \times X$ por $x \equiv y$ se $x=y$ ou $x \triangleright y$ ou $y \triangleright x$. Pelos fatos 3,5 e 6 acima, é imediato que $\equiv$ é uma relação de equivalência. Denotamos a classe de $x$ por $[x]$. Procedemos à caracterização de $(X, \sigma)$ como uma regra de escolha aleatória por categorias disjuntas.

Faça $\succ=\triangleright, \mathcal{S}=\{[x]: x \in X\}$ e $\pi([x])=p_{x}$. É claro que $\succ$ é acíclica e completa em cada categoria. Ademais, $\sum_{[x] \in \mathcal{S}} \pi([x])=\sum_{x \in X} \sigma(X, x)=1$. Por fim, segue dos fatos 1 e 4 que $\sigma(A, x)=\left\{\begin{array}{l}0, \text { se } x \notin A \text { ou } \exists y \in S_{x} \cap A \text { tal que } y \succ x \\ \pi\left(S_{x}\right), \text { caso contrário }\end{array}\right.$

\section{Conclusão}

O modelo aqui desenvolvido estende a ideia de Furtado et al. [2015], em que o tomador de decisão, por razões cognitivas, de limitação de atenção ou de racionalidade, restringe sua gama de opções a um subconjunto do universo de opções, ao paradigma das escolhas aleatórias. Assim como Furtado et al. [2015] acrescentam uma condição de aciclicidade ao modelo mais geral de pseudoracionalidade, o presente modelo acrescenta a aciclicidade ao modelo mais geral de maximização de utilidade aleatória (RUM) de Block and Marshak [1960], Marshak [1960]. Como resultado, obtemos um modelo capaz de explicar de forma bastante razoável padrões de decisão não abarcados por outros modelos disponíveis na literatura. Trata-se ao mesmo tempo de uma generalização do modelo de Manzini and Mariotti [2014]. Fica como tema para pesquisa futura a reconstrução deste e de outros modelos mais específicos que o de categorias a partir dos axiomas aqui usados.

\section{A Complemento das demonstrações}

\section{A.1 Teorema 1}

\section{A.1.1 Cálculo de $q_{\sigma}$ em função de $\sigma$.}

Dados $S \in \bar{\Omega}$ e $x \in X \backslash S$, temos a seguinte equação verificável por indução finita em $|S|$. 


$$
q_{\sigma}(S, x)=\sum_{R \subseteq S}(-1)^{|S \backslash R|} \sigma(X \backslash R, x)
$$

De fato, para $S=\emptyset$ a equação segue de imediato da construção de $q_{\sigma}$, e para $|S| \geq 1$, assumindo a hipótese de que a equação é válida para conjuntos de menor cardinalidade,

$$
\begin{aligned}
q_{\sigma}(S, x) & =\sigma(X \backslash S, x)-\sum_{R \subset S} q_{\sigma}(R, x) \\
& =\sigma(X \backslash S, x)-\sum_{R \subset S} \sum_{Q \subseteq R}(-1)^{|R \backslash Q|} \sigma(X \backslash Q, x) \\
& =\sigma(X \backslash S, x)+\sum_{Q \subset S}(-1)^{|S \backslash Q|} \sigma(X \backslash Q, x) \\
& =\sum_{R \subseteq S}(-1)^{|S \backslash R|} \sigma(X \backslash R, x)
\end{aligned}
$$

Cabe observar que

$$
\begin{aligned}
\sum_{R \subseteq S}(-1)^{|S \backslash R|} \sigma(X \backslash R, x) & =\sum_{R^{1} \subseteq S \cap U_{\succ}(x)}\left(\sum_{R^{2} \subseteq S \cap L_{\succ}(x)}(-1)^{\left|S \backslash\left(R^{1} \cup R^{2}\right)\right|} \sigma\left(X \backslash\left[R^{1} \cup R^{2}\right], x\right)\right) \\
& =\sum_{R^{1} \subseteq S \cap U_{\succ}(x)}(-1)^{\left|S \backslash R^{1}\right|}\left(\sum_{R^{2} \subseteq S \cap L_{\succ}(x)}(-1)^{\left|R^{2}\right|} \sigma\left(X \backslash\left[R^{1} \cup R^{2}\right], x\right)\right) \\
& =\sum_{R^{1} \subseteq S \cap U_{\succ}(x)}(-1)^{\left|S \backslash R^{1}\right|}\left(\sum_{R^{2} \subseteq S \cap L_{\succ}(x)}(-1)^{\left|R^{2}\right|} \sigma\left(X \backslash R^{1}, x\right)\right)
\end{aligned}
$$

Note que, se $S \cap L_{\succ}(x) \neq \emptyset$, metade dos conjuntos $R^{2} \subseteq S \cap L_{\succ}(x)$ tem cardinalidade par e metade ímpar, o que resulta em um somatório nulo. Segue portanto a afirmação que simplifica as contas mais adiante.

$$
S \cap L_{\succ}(x) \neq \emptyset \Rightarrow q_{\sigma}(S, x)=0
$$

Para a opção nula, por um método análogo àquele usado para provar 1 chega-se a 


$$
q_{\sigma}(S, \text { null })=\sum_{R \subseteq S}(-1)^{|S \backslash R|} \sum_{x \in X \backslash R} \sigma(X \backslash R, \text { null })
$$

\section{A.1.2 Cálculo de $\sigma$ em função de $q_{\sigma}$.}

Fixe $x \in X$. Defina $\bar{\Omega}_{x}=\{X \backslash A: x \in A \in \Omega\}, q_{x} \in \mathbb{R}^{\bar{\Omega}_{x}}$ dada por $q_{x}(S)=$ $q_{\sigma}(S, x)$ e $s_{x} \in \mathbb{R}^{\bar{\Omega}_{x}}$ dada por $s_{x}(S)=\sigma(X \backslash S, x)$. Considere o Poset $\left(\Omega_{x}, \supseteq\right)$, associado à função de Möbius

$$
\mu(A, B)=\left\{\begin{array}{l}
(-1)^{|A \backslash B|} \text { se } A \subseteq B \\
0 \text { caso contrário }
\end{array}\right.
$$

Pelo Teorema da Inversão de Möbius (veja Stuhlsatz),

$$
q_{x}(S)=\sum_{R \subseteq S} \mu(R, S) \sigma_{x}(R) \Leftrightarrow \sigma_{x}(S)=\sum_{R \subseteq S} q_{\sigma}(R)
$$

A equação 1 portanto é equivalente a

$$
\sigma(A, x)=\sum_{R \subseteq X \backslash A} q_{\sigma}(R, x)
$$

Definindo $\sigma_{\text {null }}$ e $q_{\text {nulll }}$ de maneira análoga, com $\bar{\Omega}_{\text {null }}=\bar{\Omega}$, pelo mesmo raciocínio a equação 3 é equivalente a

$$
\sigma(A, n u l l)=\sum_{R \subseteq X \backslash A} q_{\sigma}(R, n u l l)
$$

\section{A.1.3 $[\Rightarrow]$ Cálculo de $q_{\sigma}$ em função de $\pi$.}

Se $S \cap L_{\succ}(x) \neq \emptyset$, já vimos que $q_{\sigma}(S, x)=0$. Se $S \cap L_{\succ}(x)=\emptyset$, substituindo a função $\sigma$ na equação 1 pela representação, temos 


$$
\begin{aligned}
q_{\sigma}(S, x)= & \sum_{R \subseteq S}(-1)^{|S \backslash R|} \sigma(X \backslash R, x) \\
= & \sum_{R \subseteq S}(-1)^{|S \backslash R|} \sum_{Q \subseteq R \cup L_{\succ}(x)} \pi(Q \cup\{x\}) \\
= & \sum_{R \subseteq S}(-1)^{|S \backslash R|} \sum_{Q^{1} \subseteq R} \sum_{Q^{2} \subseteq L(x)} \pi\left(Q^{1} \cup Q^{2} \cup\{x\}\right) \\
= & \sum_{Q^{2} \subseteq L(x)} \sum_{Q^{1} \subseteq A}\left(\mid\left\{R \subseteq S: Q^{1} \subseteq R \text { e }|\{S \backslash R\}| \text { é par }\right\} \mid\right. \\
& \left.-\mid\left\{R \subseteq S: Q^{1} \subseteq R \text { e }|\{S \backslash R\}| \text { é ímpar }\right\} \mid\right) \pi\left(Q^{1} \cup Q^{2} \cup\{x\}\right)
\end{aligned}
$$

Note que

$\mid\left\{R \subseteq S: Q^{1} \subseteq R\right.$ e $|\{S \backslash R\}|$ é par $\}|-|\left\{R \subseteq S: Q^{1} \subseteq R\right.$ e $|\{S \backslash R\}|$ é ímpar $\} \mid=\left\{\begin{array}{l}1 \text { se } Q^{1}=A \\ 0 \text { se } Q^{1} \subset A\end{array}\right.$

Eliminando os termos nulos da soma, resulta que $q_{\sigma}(S, x)=\sum_{R \subseteq L_{\succ}(x)} \pi(S \cup$ $R \cup\{x\})$. Reunem-se os dois casos na afirmação abaixo.

$$
q_{\sigma}(S, x)=\left\{\begin{array}{l}
0 \text { se } S \cap L_{\succ}(x) \neq \emptyset \\
\sum_{R \subseteq L_{\succ}(x)} \pi(S \cup R \cup\{x\}) \text { caso contrário }
\end{array}\right.
$$

Para encontrar $q_{\sigma}(S$, null $)$, é necessário primeiro calcular $\sigma(A$, null $)$ em termos de $\pi$. Considere o somatório

$$
\begin{aligned}
\sum_{x \in A} \sigma(A, x) & =\sum_{x \in A} \sum_{R \subseteq[X \backslash A] \cup L_{\succ}(x)} \pi(R \cup\{x\}) \\
& =\sum_{x \in A} \psi_{x}
\end{aligned}
$$

em que $\psi_{x}=\sum_{R \subseteq[X \backslash A] \cup L_{\succ}(x)} \pi(R \cup\{x\})$. Note que, para qualquer $C \in \Omega$, se $C \cap A \neq \emptyset$, o termo $\pi(C)$ aparecerá exatamente uma vez no somatório, em $\psi_{x_{c}}$, em que $x_{c}$ é o $\succ$-máximo de $C \cap A$. Por outro lado, se $C \cap A=\emptyset, \pi(C)$ não aparece no somatório. Resulta que $\sum_{x \in A} \sigma(A, x)=\sum_{R \in \Omega: R \cap A \neq \emptyset} \pi(R)$ e 
portanto

$$
\sigma(A, n u l l)=\sum_{R \subseteq X \backslash A} \pi(R)
$$

Finalmente,

$$
\begin{aligned}
q_{\sigma}(S, \text { null })= & \sum_{R \subseteq S}(-1)^{|S \backslash R|} \sigma(X \backslash R, \text { null }) \\
= & \sum_{R \subseteq S}(-1)^{|S \backslash R|} \sum_{Q \subseteq R} \pi(Q) \\
= & \sum_{Q \subseteq S}(\mid\{R \subseteq S: Q \subseteq R \text { e }|\{S \backslash R\}| \text { é par }\} \mid \\
& -\mid\{R \subseteq S: Q \subseteq R \text { e }|\{S \backslash R\}| \text { é ímpar }\} \mid) \pi(Q)
\end{aligned}
$$

Como no caso anterior,

$\mid\{R \subseteq S: Q \subseteq R$ e $|\{S \backslash R\}|$ é par $\}|-|\{R \subseteq S: Q \subseteq R$ e $|\{S \backslash R\}|$ é ímpar $\} \mid=\left\{\begin{array}{l}1 \text { se } Q=S \\ 0 \text { se } Q \subset S\end{array}\right.$

Eliminando os termos nulos da soma, resulta que

$$
q_{\sigma}(S, n u l l)=\pi(S)
$$

A.1.4 $[\Rightarrow] \triangleright \subseteq \succ$.

Sejam $x, y \in X$ tais que $x \triangleright y$. Para algum $A \in \Omega \sigma(A, y)>\sigma(A \cup\{x\}, y)$, isto é,

$$
\sum_{R \subseteq[X \backslash A] \cup L_{\succ}(y)} \pi(R \cup\{y\})>\sum_{R \subseteq[X \backslash A \cup\{x\}] \cup L_{\succ}(y)} \pi(R \cup\{y\})
$$

Isso implica que $x \notin L_{\succ}(y)$ e que para algum $R \subseteq[X \backslash A] \cup L_{\succ}(y) x \in R$ e $\pi(R \cup\{y\})>0$. Como $\succ$ é completa na categoria $B \cup\{y\}$ e $x \notin L_{\succ}(y)$, isso 
implica que $x \succ y$.

\section{A.1.5 $[\Leftarrow]$ Cálculo de $\pi$ em função de $q_{\sigma}$.}

Sejam $S \in \mathcal{S}^{(k)}$ e $S^{\prime}=S \backslash\left\{x_{k}\right\}$. Temos

$$
\begin{aligned}
\pi(S) & =q_{\sigma}\left(S^{\prime}, x_{k}\right)-\sum_{i=k+1}^{n} q_{\sigma}\left(S, x_{i}\right) \\
& =\sum_{R \subseteq S^{\prime}}(-1)^{\left|S^{\prime} \backslash R\right|} \sigma\left(X \backslash R, x_{k}\right)-\sum_{i=k+1}^{n} \sum_{R \subseteq S}(-1)^{|S \backslash R|} \sigma\left(X \backslash R, x_{i}\right) \\
& =\sum_{R \subseteq S^{\prime}}(-1)^{\left|S^{\prime} \backslash R\right|} \sigma\left(X \backslash B, x_{k}\right)-\sum_{i=k+1}^{n} \sum_{R \subseteq S^{\prime}}(-1)^{\left|S^{\prime} \backslash R\right|}\left(\sigma\left(X \backslash R, x_{i}\right)-\sigma\left(X \backslash\left[R \cup\left\{x_{k}\right\}\right], x_{i}\right)\right) \\
& =\sum_{R \subseteq S^{\prime}}(-1)^{\left|S^{\prime} \backslash R\right|}\left(\sum_{i=k}^{n} \sigma\left(X \backslash R, x_{i}\right)-\sum_{i=k}^{n} \sigma\left(X \backslash\left[R \cup\left\{x_{k}\right\}\right], x_{i}\right)\right)
\end{aligned}
$$

em que no último passo usamos o fato de que $\sigma\left([X \backslash R] \backslash\left\{x_{k}\right\}, x_{k}\right)=0$.

Note que para todo $i<k \sigma\left([X \backslash R], x_{i}\right)-\sigma\left([X \backslash R] \backslash\left\{x_{k}\right\}, x_{i}\right)=0$, já que $x_{k} \not x_{i}$. Podemos então completar o somatório acima com os termos $\sum_{i=1}^{k-1} \sigma\left([X \backslash R], x_{i}\right)-\sum_{i=1}^{k-1} \sigma\left([X \backslash R] \backslash\left\{x_{k}\right\}, x_{i}\right)$, donde resulta que

$$
\begin{aligned}
\pi(S) & =\sum_{R \subseteq S^{\prime}}(-1)^{\left|S^{\prime} \backslash R\right|}\left(\sum_{i=1}^{n} \sigma\left([X \backslash R], x_{i}\right)-\sum_{i=1}^{n} \sigma\left([X \backslash R] \backslash\left\{x_{k}\right\}, x_{i}\right)\right) \\
& =\sum_{R \subseteq S^{\prime}}(-1)^{\left|S^{\prime} \backslash R\right|}\left((1-\sigma([X \backslash R], \text { null }))-\left(1-\sigma\left([X \backslash R] \backslash\left\{x_{k}\right\}, \text { null }\right)\right)\right) \\
& =\sum_{R \subseteq S^{\prime}}(-1)^{\left|S^{\prime} \backslash R\right|}\left(\sigma\left([X \backslash R] \backslash\left\{x_{k}\right\}, \text { null }\right)-\sigma([X \backslash R], \text { null })\right) \\
& =\sum_{R \subseteq S}(-1)^{|S \backslash R|} \sigma([X \backslash R], \text { null }) \\
& =q_{\sigma}(S, \text { null })
\end{aligned}
$$

\section{A.1.6 $[\Leftarrow]$ Cálculo de $\sigma$ em função de $\pi$}

Tome um $R \subseteq\left\{x_{1}, \ldots, x_{k-1}\right\}$ qualquer. A função $\pi$ foi construída de forma que, nesse domínio, 


$$
q_{\sigma}\left(R, x_{k}\right)=\sum_{Q \subseteq\left\{x_{k+1}, \ldots, x_{n}\right\}} \pi\left(R \cup Q \cup\left\{x_{k}\right\}\right)
$$

De fato, para $l \in\{k+1, \ldots, n\}$, se $Q \subseteq\left\{x_{k+1, \ldots,}, x_{l-1}\right\}$, teremos por construção $\pi\left(R \cup Q \cup\left\{x_{k}\right\}\right)=q_{\sigma}\left(R \cup Q \cup\left\{x_{k}\right\}, x_{i}\right)-\sum_{i=l+1}^{n} q_{\sigma}\left(R \cup Q \cup\left\{x_{k}, x_{i}\right\}\right)$. Assim,

$$
\begin{aligned}
\sum_{Q \subseteq\left\{x_{k+1}, \ldots, x_{n}\right\}} \pi\left(R \cup Q \cup\left\{x_{k}\right\}\right)= & q_{\sigma}\left(R, x_{k}\right)+\sum_{i=k+1}^{n} \sum_{Q \subseteq\left\{x_{k+1}, \ldots, x_{i-1}\right\}}\left(q_{\sigma}\left(R \cup Q \cup\left\{x_{k}\right\}, x_{i}\right)\right. \\
& \left.-\sum_{j=i+1}^{n} q_{\sigma}\left(R \cup Q \cup\left\{x_{k}, x_{i}\right\}, x_{j}\right)\right)
\end{aligned}
$$

Note que, no somatório acima, dados $l \in\{k+1, \ldots, n\}$ e $Q \subseteq\left\{x_{k+1}, \ldots, x_{l-1}\right\}$, o termo $q_{\sigma}\left(R \cup Q, x_{l}\right)$ será subtraído exatamente uma vez, na iteração $\pi(R \cup Q \cup$ $\left.\left\{x_{k}\right\}\right)$, e somado exatamente uma vez, na iteração $\pi\left(R \cup Q \cup\left\{x_{k}, x_{l}\right\}\right)$. Anulando esses termos para todos $l$ e $C$, resulta a equação 10 .

Procedemos agora ao cálculo de $\sigma$ em função de $\pi$. Sejam $A \in \Omega, x_{k} \in A$ e $\bar{A}=A \cup\left\{x_{k+1}, \ldots, x_{n}\right\}$. Note que $\sigma\left(A, x_{k}\right)=\sigma\left(\bar{A}, x_{k}\right)$, caso contrário teríamos $x_{l} \triangleright x_{k}$ para algum $l>k$. Temos por A.1.2

$$
\sigma\left(A, x_{k}\right)=\sigma\left(\bar{A}, x_{k}\right)=\sum_{R \subseteq X \backslash \bar{A}} q_{\sigma}\left(R, x_{k}\right)
$$

Substituindo $q_{\sigma}\left(R, x_{k}\right)$ conforme 10 , encontramos

$$
\begin{aligned}
\sigma\left(A, x_{k}\right) & =\sum_{R \subseteq X \backslash \bar{A}}\left(\sum_{Q \subseteq\left\{x_{k_{+1}, \ldots, x_{n}}\right\}} \pi\left(R \cup Q \cup\left\{x_{k}\right\}\right)\right) \\
& =\sum_{R \subseteq[X \backslash A] \cup\left\{x_{k_{+1}, \ldots, x_{n}}\right\}} \pi\left(R \cup\left\{x_{k}\right\}\right)
\end{aligned}
$$

em que usamos o fato de que $(X \backslash \bar{A}) \cap\left\{x_{k+1}, \ldots, x_{n}\right\}=\emptyset$.

\section{A.1.7 $[\Leftarrow]$ Cálculo de $\sum_{S \in \mathcal{S}} \pi(S)$}

Deriva-se de 11 o somatório 


$$
\sum_{x \in X} \sigma(X, x)=\sum_{x \in X} \sum_{R \subseteq L_{\succ}(x)} \pi(R \cup\{x\})
$$

Note que cada $S=R \cup\{x\} \in 2^{X} \backslash \emptyset$ é somado exatamente uma vez, na iteração em que $x=\max _{\succ}(S)$ e $R=S \backslash \max _{\succ}(S) \subseteq L_{\succ}(x)$. Isso implica que

$$
\sum_{S \in 2^{X} \backslash \emptyset} \pi(S)=\sum_{x \in X} \sigma(X, x)=1
$$

\section{A.2 MM - caso especial}

Dada a representação de $\sigma$ por categorias, temos

$$
\begin{aligned}
\sigma(A, x) & =\sum_{S \subseteq[X \backslash A] \cup L_{\succ}(x)} \pi(S \cup\{x\}) \\
& =\sum_{S \subseteq[X \backslash A] \cup L_{\succ}(x)} \prod_{w \in S \cup\{x\}} \gamma(w) \prod_{z \notin S \cup\{x\}}(1-\gamma(z))
\end{aligned}
$$

Fazendo $C=[X \backslash A] \cup L_{\succ}(x)$, e pondo em evidência os termos que não dependem do somatório,

$$
\sigma(A, x)=\left(\gamma(x) \prod_{y \in X \backslash C}(1-\gamma(y))\right) \sum_{S \subseteq C} \prod_{w \in S} \gamma(w) \prod_{z \notin S}(1-\gamma(z))
$$

Note porém que $\sum_{S \subseteq C} \prod_{w \in S} \gamma(w) \prod_{z \notin S}(1-\gamma(z))=1$. Observando que $X \backslash C=A \cap U_{\succ}(x)$, resulta que

$$
\sigma(A, x)=\gamma(x) \prod_{y \in A \cap U_{\succ}(x)}(1-\gamma(y))
$$




\section{A.3 Teorema 2}

A.3.1 Para todos $A \in \Omega$ e $x \in X \sigma(A, x)=\left\{\begin{array}{l}0, \text { se } x \notin A \text { ou } \exists y \in A: y \triangleright x \\ p_{x}, \text { caso contrário }\end{array}\right.$

Se $x \notin A$, por definição $\sigma(A, x)=0$. Se $x \in A$, suponha que $\sigma(A, x) \neq p_{x}$. Então deve existir um $A^{\prime}$ minimal satisfazendo $x \in A^{\prime} \subseteq A$ e $\sigma\left(A^{\prime}, x\right) \neq p_{x}$. Claramente $\{x\} \neq A^{\prime}$. Tome qualquer $y \in A^{\prime} \backslash\{x\}$. Pela minimalidade de $A^{\prime}$, $\sigma\left(A^{\prime} \backslash\{y\}, x\right)=p_{x} \neq \sigma\left(A^{\prime}, x\right)$, donde, por p-Independência, $\sigma\left(A^{\prime}, x\right)=0$ e daí $y \triangleright x$. Ora, $y \in A$, o que implica novamente por p-Independência que $\sigma(A, x)=0$. Conclui-se que $[x \notin A$ e $\sigma(A, x)=0]$ ou $\left[\sigma(A, x)=p_{x}\right]$ ou $[\exists y \in A: y \triangleright x$ e $\sigma(A, x)=0]$, o que equivale à afirmação.

A.3.2 (Monotonicidade) Para todos $A, B \in \Omega_{X}$ com $A \subseteq B, \forall x \in$ $A \sigma(A, x) \geq \sigma(B, x)$ e $\sigma(A$, null $) \geq \sigma(B$, null $)$.

Primeiro note que, dados $A \in \Omega$ e $x \in X$, se para todo $y \in A \sigma(A, y)=$ $\sigma(A \cup\{x\}, y)$ então $\sigma(A$, null $)=1-\sum_{y^{\prime} \in A} \sigma\left(A, y^{\prime}\right) \geq 1-\sum_{y^{\prime} \in A} \sigma\left(A, y^{\prime}\right)-$ $\sigma(A \cup\{x\}, x)=1-\sum_{y^{\prime} \in A x} \sigma\left(A \cup\{x\}, y^{\prime}\right)=\sigma(A \cup\{x\}$, null $)$. Se por outro lado $\sigma(A, y) \neq \sigma(A \cup\{x\}, y)$ para algum $y \in A$, então por p-Independência $\sigma(A, n u l l)=\sigma(A \cup\{x\}$, null $)$. Em qualquer caso, $\sigma(A, n u l l) \geq \sigma(A \cup\{x\}$, null $)$. Portanto, para todo $A, B \in \Omega \operatorname{com} A \subseteq B$, um número finito de passos mostra que $\sigma(A$, null $) \geq \sigma(B$, null $)$. Agora, seja $x \in A \subseteq B$. Pela Afirmação 1, basta provar que $\sigma(A, x)=0 \Rightarrow \sigma(B, x)=0$. Se $\sigma(A, x)=0$, de modo similar ao caso null acima, deve existir um $A^{\prime}$ minimal com $x \in A^{\prime} \subseteq A$ tal que $\sigma\left(A^{\prime}, x\right)=0$. Sabemos que $\{x\} \neq A^{\prime}$, portanto podemos escolher $z \in A^{\prime} \backslash\{x\}$ e temos $\sigma\left(A^{\prime} \backslash\{z\}, x\right)=P_{x} \neq \sigma\left(A^{\prime}, x\right)$ e portanto, por p-Independência, como $z \in A, \sigma(B, x)=0$.

\section{A.3.3 Propriedades de $\triangleright$.}

1. (Transitividade) $x \triangleright y \triangleright z \Rightarrow x \triangleright z$.

Por Monotonicidade, temos $\sigma(\{x, y, z\}, y)=\sigma(\{x, y, z\}, z)=0, \log \sigma \sigma(\{x, y, z\}, x)+$ $\sigma(\{x, y, z\}$, null $)=1$. Então, $\sigma(\{x, z\}, z)=1-[\sigma(\{x, z\}, x)+\sigma(\{x, z\}$, null $)] \leq$ $1-[\sigma(\{x, y, z\}, x)+\sigma(\{x, y, z\}, n u l l)]=0$. Finalmente, como $\sigma(\{z\}, z)=$ $p_{z} \neq 0$ e $\sigma(\{x, z\}, z)=0$, por definição $x \triangleright z$.

2. $x \triangleright y \Rightarrow p_{x}=p_{y}$. 
Por p-Independência, $\sigma(\{x, y\}, y)=0$ e $\sigma(\{x, y\}$, null $)=\sigma(\{y\}$, null $)=$ $1-p_{y}$. Portanto, $\sigma(\{x, y\}, x)=p_{y}$. E ainda, pela Afirmação $1, \sigma(\{x, y\}, x) \neq$ $0 \Rightarrow \sigma(\{x, y\}, x)=p_{x}$.

3. $x \triangleright y, z \Rightarrow y \triangleright z$ ou $z \triangleright y$.

Por 1 , sabemos que $p_{x}=p_{y}=p_{z}$. Chame $p$ esse valor. Sabemos também por p-Independência que $\sigma(\{x, y, z\}, y)=\sigma(\{x, y, z\}, z)=0$ e daí $\sigma(\{x, y, z\}, x)=p$, e que $\sigma(\{y, z\}, n u l l)=\sigma(\{x, y, z\}$, null $)=1-p$. Portanto, $\sigma(\{y, z\}, y)+\sigma(\{y, z\}, z)=p$. Finalmente, pela Afirmação 1, $\sigma(\{y, z\}, y)=0$ ou $\sigma(\{y, z\}, z)=0$.

4. $x, y \triangleright z \Rightarrow x \triangleright y$ ou $y \triangleright x$.

Novamente temos $p_{x}=p_{y}=p_{z}$. Por p-Independência, $\sigma(\{x, y, z\}, z)=0$ e $\sigma(\{x, y, z\}, n u l l)=\sigma(\{x, z\}$, null $)=1-p$. Então temos que ter exatamente um dos valores $\sigma(\{x, y, z\}, x)$ e $\sigma(\{x, y, z\}, y)$ igual a $p$, o outro sendo necessariamente zero. Isso implica que $\sigma(\{x, y\}, x)=0$ ou $\sigma(\{x, y\}, y)=0$, caso contrário teríamos uma contradição com p-Independência. Concluimos que $x \triangleright y$ ou $y \triangleright x$.

\section{Referências}

Salvador Barberá and Prasanta K. Pattanaik. Falmagne and the rationalizability of stochastic choices in terms of random orderings. Econometrica, 1986.

H.D. Block and J. Marshak. Random orderings and stochastic theories of responses. Olkin, I., S.G. Gurye, W. Hoeffding, W.G. Madow and H.B. Mann (eds.) Constributions do Probability and Statistics, Stanford University Press, Stanford, CA., 1960.

J.C. Falmagne. A representation theorem for finite random scale systems. Journal of Mathematical Psychology, 1978.

Bruno A. Furtado, Leandro Nascimento, and Gil Riella. Rational choice with categories. mimeo, Universidade de Brasília, 2015.

Faruk Gul, Paulo Natenzon, and Wolfgang Pesendorfer. Random choice as behavioral optimization. Econometrica, 2014.

Robert Duncan Luce. Individual choice behavior, a theoretical analysis. Wiley: New York, 1959. 
Paola Manzini and Marco Mariotti. Stochastic choice and consideration sets. Econometrica, 82(3):1153-1176, 2014.

J. Marshak. Binary choice constraints and random utility indicators. Cowles Foundation, 1960.

Paul Samuelson. A note on the pure theory of consumers behavior. Econometrica, 1938.

Erin Stuhlsatz. Möbius inversion formula. https://www.whitman.edu/Documents/Academics/Mathematics/stuhlsatz.pdf. 